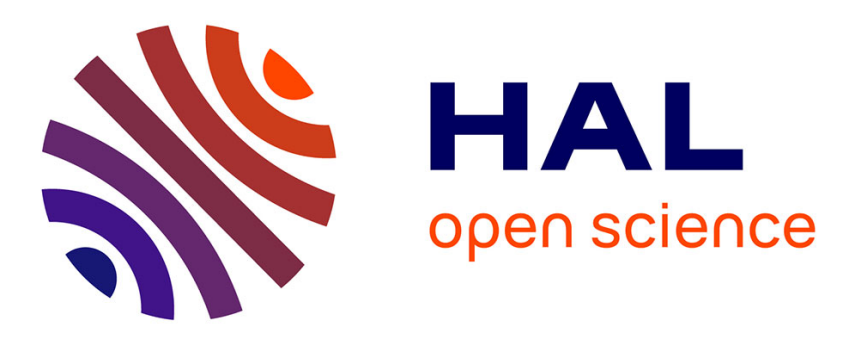

\title{
Constant modulus hybrid beamforming for multi-user systems in the presence of blockers
}

Mohammed Reda Bekkar, Benoit Miscopein, Serge Bories, Laurent Ros, Cyrille Siclet

\section{To cite this version:}

Mohammed Reda Bekkar, Benoit Miscopein, Serge Bories, Laurent Ros, Cyrille Siclet. Constant modulus hybrid beamforming for multi-user systems in the presence of blockers. ICT 2018 - 25th International Conference on Telecommunications, Jun 2018, Saint-Malo, France. hal-01835898

\section{HAL Id: hal-01835898 https://hal.science/hal-01835898}

Submitted on 11 Jul 2018

HAL is a multi-disciplinary open access archive for the deposit and dissemination of scientific research documents, whether they are published or not. The documents may come from teaching and research institutions in France or abroad, or from public or private research centers.
L'archive ouverte pluridisciplinaire $\mathbf{H A L}$, est destinée au dépôt et à la diffusion de documents scientifiques de niveau recherche, publiés ou non, émanant des établissements d'enseignement et de recherche français ou étrangers, des laboratoires publics ou privés. 


\title{
Constant modulus hybrid beamforming for multi-user systems in the presence of blockers
}

\author{
Mohammed Reda Bekkar ${ }^{\dagger \ddagger}$, Benoit Miscopein ${ }^{\dagger}$, Serge Bories ${ }^{\dagger}$, Laurent Ros ${ }^{\ddagger}$ Cyrille Siclet $\ddagger$ \\ mohammed-reda.bekkar@cea.fr, benoit.miscopein@cea.fr, serge.bories@cea.fr \\ laurent.ros@gipsa-lab.fr cyrille.siclet@gipsa-lab.fr \\ ${ }^{\dagger}$ CEA-LETI, Minatec campus, Grenoble, France \\ ${ }^{\ddagger}$ Univ. Grenoble Alpes, CNRS, Grenoble INP*, GIPSA-lab, 38000 Grenoble, France \\ * Institute of Engineering Univ. Grenoble Alpes
}

\begin{abstract}
Hybrid beamforming (HBF) solutions, involving both analog and digital stages, are considered as key enablers for next generation of small cell networks wireless communications. They provide interference rejection and spatial multiplexing features for the base stations with fewer radio frequency (RF) chains and analog-to-digital converters (ADCs) compared to the optimal full digital beamforming. However jointly designing the digital beamforming matrix and the analog beamforming matrix with constant modulus entries makes the optimal signal-to-interferenceplus-noise ratio (SINR) solution complex to find. In this paper we propose a simple solution and investigate its SINR loss as a function of angle of arrivals of blockers. The solution consists of setting the analog weights phases equal to those of a ful digital beamformer and keeping the modulus of the weights constant, while the complementary digital part is designed with respect to the effective channel matrix. This algorithm is applied over different full digital algorithms, in a medium signal-to-noise ratio (SNR) and high blocker levels. We show that despite that the analog stage introduces losses, the digital stage is still able to compensate them almost to the performance of the optimal full digital beamforming, and so with minor difference between algorithms used in analog stage.
\end{abstract}

\section{INTRODUCTION}

Due to the projected data rate demand increase in 2020 by a 1000 factor for the aggregate data rate networks [1], the next generation of mobile networks will have to operate in ultra user-dense scenarios. Key enablers proposed in the literature and by industrials to support this demand are the small cell networks (SCNs) [2] that will operate in short range with a prefered TDD (time division duplex) based access, due to the difficulty of coordination between base stations in such scenarios, the channel reciprocity that can be used to reduce feedback overhead and the simpler RF front-end structure [1].

However a principal drawback of the SCNs is a low SINR operating regime due to the high amount of co-users and co-base stations interference. A typical scenario where this problem happens is depicted in Fig. 1, two small cells (SCs) operating in TDD asynchronously with the one in downlink interfere with the adjacent one in uplink. This puts a bottleneck on the network capacity as well as the simultaneously supported number of users especially if the deployment inter-site distance (ISD) is further decreased. As a response to this problem the introduction of a multi-antenna processing system on the PHY-
Layer is of high interest in the recent literature [3], [4], [5], [6], [7], [8].

The use of millimeter waves $(\mathrm{mmW})$ frequency bands will make the implementation of a high number of antennas possible in multiple-input multiple-output (MIMO) systems, which will help combat the high pathloss present in these bands as well as enable a high simultaneously-supported number of users, especially in massive MIMO scenarios. However the cost, energy consumption and a high sensitivity of the ADCs to blockers [5] of such systems make it unaffordable and too complex [6] in the case of full digital implementation, especially as the number of antennas grows, and so the other RF components (RF chains, mixers etc) which scale with the same number. As a converse implementation method, full analog solution could be envisioned, to spatially filter the blockers before digital conversion, nonetheless its main disadvantages are the sensitivity to characteristics drift and the lack of flexibility. Although in centimeter waves $(\mathrm{cmW})$ and microwave frequency bands MIMO systems will not allow the use of a high number of antennas as compared to the $\mathrm{mmW}$ bands, these frequencies do not suffer from a high path-loss and channel models and RF components are readily available. Recent studies explored new strategies to alleviate the aforementioned problems, namely to reduce the cost and the consumption of the full digital architecture. One promising way is to reduce ADCs resolution [9] to decrease the system power consumption. Another promising way, is to mix the two architectures (analog and digital) into a hybrid one to take advantage of each architecture type [3], [4], [5], [6], [7], [8], and relieve their respective drawbacks.

Hybrid beamforming (HBF) allows the use of fewer RF chains and ADCs (which scale with the number of users only) as compared to the full digital architecture, also it allows the spatial filtering of blockers [5] through the analog stage, while the lower dimensional digital stage will refine the interference rejection, and separate users. With this strategy it is possible to approach the full digital performances.

In HBF different analog circuitry complexities are available and influence the analog beamweights computation complexity. The main differences reside in the number of RF chains connected to the antenna array (fully connected architecture, or partially connected architecture [3]) and beamweights imple- 
mentation devices, namely using phase shifters and amplifiers (which allow the weights choice in $\mathbb{C}^{n_{B S} \times 1}$ with $n_{B S}$ antennas) or phase shifters only (which restricts the choice to the set of vectors with constant modulus entries $\mathcal{C M}=\left\{\mathbf{a} \in \mathbb{C}^{n_{B S} \times 1}\right.$ : $\left.\left.\left|[\mathbf{a}]_{i}\right|=1 \quad \forall i=1 \ldots n_{B S}\right\}\right)$.

HBF has been adressed in several previous works with different channel models, channel state information assumptions, cost functions to optimize, analog front end architecture and multiuser/single-user scenarios. In this work we will focus on uplink beamforming solutions for SCs with a simple single path $\mathrm{cmW}$ channel model and perfect channel state information knowledge at the base station receiver side. In this paper we will focus on analog beamweights using phase shifters only. This constraint makes the feasible region non-convex, and as a result the analog stage optimal solution in terms of SINR NP-Hard to find [5]. Thus joint design of the analog and digital stages is difficult. Separate analog and digital beamforming matrices design has been adressed in [8]. The authors address a multi-user scenario and propose a solution based on Gram-Schmidt method to design complex beamweights first. Second, they design the analog beamforming matrix with a simple scheme by taking the beamweights phases of the full digital solution. The digital stage is then designed through the minimum mean-square error (MMSE) algorithm. In [5] the authors address a multi-user scenario with blockers and propose a solution with a user-toblockers SINR optimal solution in the phase constrained analog stage. The MMSE algorithm is then used in the digital part. Various full digital solutions have been also explored in similar scenarios, for e.g. in [10] where zero forcing (ZF), maximumratio combining (MRC) and MMSE algorithms are compared in terms of energy and spectral efficiency. In [8] the authors introduced a simple scheme for analog stage design which consists of plainly setting the weights to Gram-Schmidt full digital weights with a modulus equal to one. In this paper we generalize this scheme based on two other algorithms namely $\mathrm{ZF}$ and Capon. Our objective is to assess performance loss as compared to the full digital solution. To do so we compute an SINR output of different solutions versus blocker angle of arrival (AoA) as in [11], which will allow us to visualize spatial behaviour of the algorithms.

In section II we present the system signals and model. In section III computer simulations results are given to analyze the SINR variation as a function of the angle of arrival of an interferer. Conclusions are drawn in section IV.

\section{SYSTEM AND SIGNAL MODEL}

Capital boldface characters are used to note matrices, and lower case boldface characters are used to note column vectors. The $N \times N$ identity matrix is noted $\mathbf{I}_{N} \cdot(\cdot)^{H},(\cdot)^{-1}$ denote the conjugate transpose and the matrix inverse respectively. The $(k, m)$-th entry of a matrix $\mathbf{A}$ is noted $[\mathbf{A}]_{k, m}$ and its $(k)$-th column $\mathbf{a}^{(k)}$. The element-wise exponentiation and the element-wise phases of a matrix $\mathbf{A}$ are noted $e^{\mathbf{A}}$ and $\arg (\mathbf{A})$ respectively.

Consider $n_{U}$ single antenna users and $n_{B}$ single antenna blockers transmitting to an $n_{B S}$ antennas base station. The

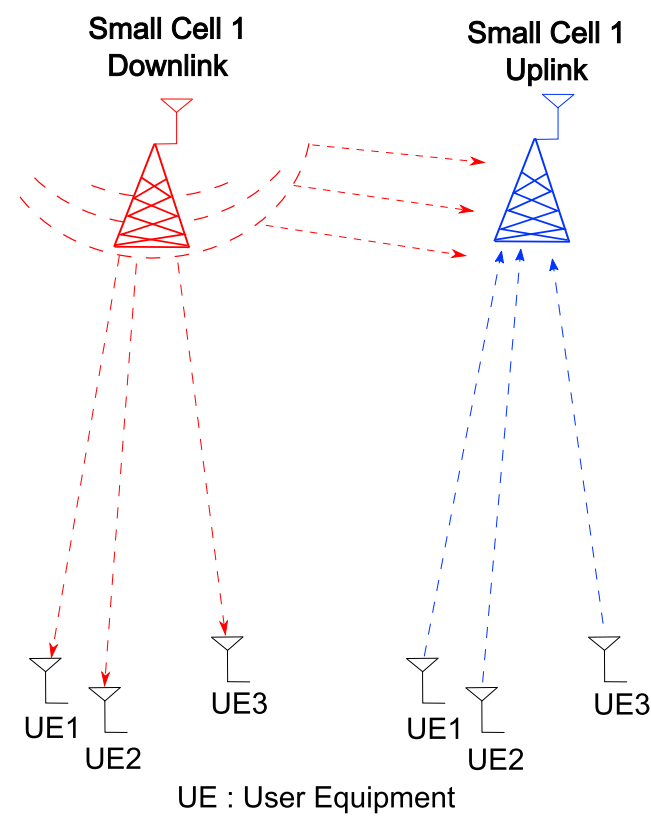

Fig. 1. Typical interference scenario between two adjacent small cells operating in TDD. The small cell 1 operating in downlink is jamming small cell 2 operating in uplink.

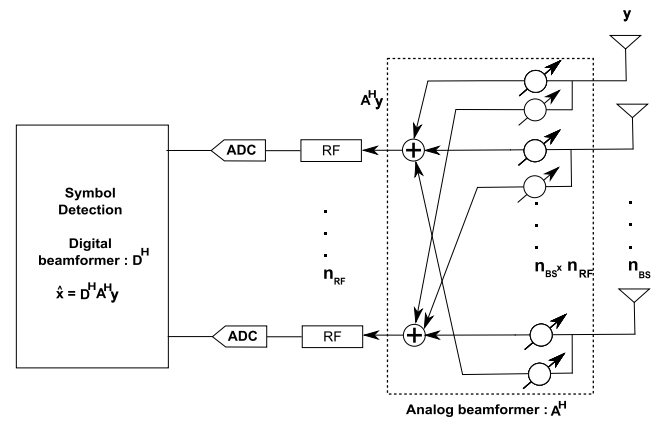

Fig. 2. Full complexity hybrid beamformer architecture.

received signal at the base station in the case of non-frequency selective channel model is as follows :

$$
\mathbf{y}=\mathbf{H} \mathbf{x}+\mathbf{H}_{B} \mathbf{x}_{B}+\mathbf{n}
$$

Where $\mathbf{y} \in \mathbb{C}^{n_{B S} \times 1}$ is the received signal vector, $\mathbf{x} \in \mathbb{C}^{n_{U} \times 1}$ is the users transmitted signal vector, $\mathbf{x}_{B} \in \mathbb{C}^{n_{B} \times 1}$ is the blockers signal vector, $\mathbf{n} \in \mathbb{C}^{n_{B S} \times 1}$ is the thermal noise vector, with $[\mathbf{n}]_{i} \sim \mathcal{C} \mathcal{N}\left(0, \sigma_{n}^{2}\right), \mathbf{H}=\left[\mathbf{h}^{(1)} \mathbf{h}^{(2)} \ldots \mathbf{h}^{\left(n_{U}\right)}\right] \in$ $\mathbb{C}^{n_{B S} \times n_{U}}$ is the users attenuation channel matrix, and $\mathbf{H}_{B}=$ $\left[\mathbf{h}_{B}^{(1)} \mathbf{h}_{B}^{(2)} \ldots \mathbf{h}_{B}^{\left(n_{B}\right)}\right] \in \mathbb{C}^{n_{B S} \times n_{B}}$ is the blockers attenuation channel matrix (with a similar structure to $\mathbf{H}$.) In this work, we assume perfect knowledge of the channel matrices.

For a user of interest $k(1)$ can be rewritten:

$$
\mathbf{y}=\mathbf{h}^{(k)} x^{(k)}+\sum_{\substack{i=1 \\ i \neq k}}^{n_{U}} \mathbf{h}^{(i)} x^{(i)}+\sum_{i=1}^{n_{B}} \mathbf{h}_{B}^{(i)} x_{B}^{(i)}+\mathbf{n}
$$

We can notice that a user of interest's signal is carried by its spatial signature $\mathbf{h}^{(k)}$, and the terms $\mathbf{h}^{(i)} x_{i}$ and $\mathbf{h}_{B}^{(i)} x_{B}^{(i)}$ can be 
viewed as inter-user interference and blockers interference.

In the case of full digital beamforming, the linear processing at the base station can be modeled as :

$$
\hat{\mathbf{x}}=\mathbf{W}^{H} \mathbf{y}=\mathbf{W}^{H} \mathbf{H} \mathbf{x}+\mathbf{W}^{H}\left(\mathbf{H}_{B} \mathbf{x}_{B}+\mathbf{n}\right)
$$

Where $\mathbf{W} \in \mathbb{C}^{n_{B S} \times n_{U}}$ is the full digital beamforming matrix. For a user of interest $k$ (3) can be rewritten:

$$
\begin{aligned}
& \hat{x}^{(k)}=\mathbf{w}^{(k) H} \mathbf{h}^{(k)} x^{(k)}+ \\
& \mathbf{w}^{(k) H}\left(\sum_{\substack{i=1 \\
i \neq k}}^{n_{U}} \mathbf{h}^{(i)} x^{(i)}+\sum_{i=1}^{n_{B}} \mathbf{h}_{B}^{(i)} x_{B}^{(i)}+\mathbf{n}\right)
\end{aligned}
$$

Where $\mathbf{w}^{(k)}$ is the kth column of $\mathbf{W}$. Without loss of generality we consider unity transmitting powers for users and blockers. The SINR output $\gamma_{F D}^{(k)}$ of the $\mathbf{w}^{(k)}$ full digital spatial filter is:

$$
\gamma_{F D}^{(k)}=\frac{\left|\mathbf{w}^{(k) H} \mathbf{h}^{(k)}\right|^{2}}{\sum_{\substack{i=1 \\ i \neq k}}^{n_{U}}\left|\mathbf{w}^{(k) H} \mathbf{h}^{(i)}\right|^{2}+\sum_{i=1}^{n_{B}}\left|\mathbf{w}^{(k) H} \mathbf{h}_{B}^{(i)}\right|^{2}+\sigma_{n}^{2}\left\|\mathbf{w}^{(k)}\right\|^{2}}
$$

In the case of hybrid beamforming, the linear processing at the base station can be modeled as:

$$
\hat{\mathbf{x}}=(\mathbf{A D})^{H} \mathbf{y}=\mathbf{D}^{H} \mathbf{A}^{H} \mathbf{H} \mathbf{x}+\mathbf{D}^{H} \mathbf{A}^{H}\left(\mathbf{H}_{B} \mathbf{x}_{B}+\mathbf{n}\right)
$$

Where $\mathbf{A} \in \mathbb{C}^{n_{B S} \times n_{R F}}$ is the analog beamforming matrix, $\mathbf{D} \in \mathbb{C}^{n_{R F} \times n_{U}}$ is the digital beamforming matrix, and $n_{R F}$ is the number of RF chains.

The structure of the analog beamforming matrix A directly depends on the RF circuitry architecture; a non-zero entry $[\mathbf{A}]_{i j}$ reflects a connection between the $j$ th $\mathrm{RF}$ chain and the $i$ th antenna through an analog weight $a_{i j} e^{\phi_{i j}}$ consisting of an amplifier and a phase shifter. Since our focus is on phase shifter only architecture, we will consider that the analog beamweights are chosen in $\mathcal{C M} \Leftrightarrow a_{i j}=1 \forall i, j$. This architecture is summerized in fig. (2). The SINR output $\gamma_{A}^{(k)}$ of the $\mathbf{a}^{(k)}$ analog spatial filter is:

$$
\gamma_{A}^{(k)}=\frac{\left|\mathbf{a}^{(k) H} \mathbf{h}^{(k)}\right|^{2}}{\sum_{\substack{i=1 \\ i \neq k}}^{n_{U}}\left|\mathbf{a}^{(k) H} \mathbf{h}^{(i)}\right|^{2}+\sum_{i=1}^{n_{B}}\left|\mathbf{a}^{(k) H} \mathbf{h}_{B}^{(i)}\right|^{2}+n_{B S} \sigma_{n}^{2}}
$$

Since the digital beamformer sees the effective channel matrices $\mathbf{H}_{e}=\mathbf{A}^{H} \mathbf{H}$ and $\mathbf{H}_{B, e}=\mathbf{A}^{H} \mathbf{H}_{B}$ The SINR output $\gamma_{D}^{(k)}$ of the $\mathbf{d}^{(k)}$ digital spatial filter is:

$$
\gamma_{D}^{(k)}=\frac{\left|\mathbf{d}^{(k) H} \mathbf{h}_{e}^{(k)}\right|^{2}}{\sum_{\substack{i=1 \\ i \neq k}}^{n_{U}}\left|\mathbf{d}^{(k) H} \mathbf{h}_{e}^{(i)}\right|^{2}+\sum_{i=1}^{n_{B}}\left|\mathbf{d}^{(k) H} \mathbf{h}_{B, e}^{(i)}\right|^{2}+\sigma_{n}^{2}\left\|\mathbf{A}^{H} \mathbf{d}^{(k)}\right\|^{2}}
$$

From (6) and (3) we notice that the design of a full digital or a hybrid beamforming algorithm reduces to a design of a single matrix $\mathbf{W}$ or two matrices $\mathbf{A}$ and $\mathbf{D}$ respectively. These matrices can be designed to maximize a cost functions like the SINR or the sum rate.

\section{A. Full digital solutions}

1) Zero forcing solution: To compute the ZF solution with blockers consideration we have to rewrite the model as:

$$
\begin{aligned}
& \mathbf{y}=\left[\begin{array}{ll}
\mathbf{H} & \mathbf{H}_{B}
\end{array}\right]\left[\begin{array}{c}
\mathbf{x} \\
\mathbf{x}_{B}
\end{array}\right]+\mathbf{n} \\
& \mathbf{H}^{\prime}=\left[\begin{array}{ll}
\mathbf{H} & \mathbf{H}_{B}
\end{array}\right] \\
& \mathbf{W}_{Z F}^{\prime}=\mathbf{H}^{\prime}\left(\mathbf{H}^{\prime H} \mathbf{H}^{\prime}\right)^{-1} \\
& \mathbf{W}_{Z F}=\left[\mathbf{w}_{Z F}^{\prime(1)} \ldots \mathbf{w}_{Z F}^{\prime\left(n_{U}\right)}\right]
\end{aligned}
$$

Since with this model, (11) computes beamweights for users and blockers, we use (12) to select only the $n_{U}$ first columns of $\mathbf{W}_{Z F}$.

2) Gram-Schmidt solution: In [8] a Gram-Schmidt based solution is presented for a multi-user scenario without blockers. We present here a Gram-Schmidt solution on a multi-user scenario with blockers. As for the $\mathrm{ZF}$ solution we have to rewrite the models:

$$
\begin{aligned}
\mathbf{y} & =\left[\begin{array}{ll}
\mathbf{H}_{B} & \mathbf{H}
\end{array}\right]\left[\begin{array}{c}
\mathbf{x}_{B} \\
\mathbf{x}
\end{array}\right]+\mathbf{n} \\
\mathbf{H}^{\prime} & =\left[\begin{array}{ll}
\mathbf{H}_{B} & \mathbf{H}
\end{array}\right] \\
\mathbf{w}_{G S}^{\prime(1)} & =\mathbf{h}_{B}^{(1)} \\
\mathbf{w}_{G S}^{\prime(k)} & =\mathbf{h}^{\prime(k)}-\sum_{i=1}^{k-1} \frac{\mathbf{w}_{G S}^{\prime(k) H} \mathbf{h}^{\prime(k)}}{\left\|\mathbf{w}_{G S}^{\prime(k)}\right\|^{2}} \mathbf{w}_{G S}^{\prime(k)} \\
\mathbf{W}_{G S} & =\left[\mathbf{w}_{G S}^{\prime\left(n_{B}+1\right)} \ldots \mathbf{w}_{G S}^{\prime\left(n_{B}+n_{U}\right)}\right]
\end{aligned}
$$

Where the $\mathbf{h}^{\prime(k)}$ are the columns of $\mathbf{H}^{\prime}$. This column ordering allows interference canceling of all blockers for every user. As to inter-user interference canceling, users ordering influences the SINR of every user and the last processed user will have the highest one.

3) Capon solution: The optimal solution in terms of SINR is a well known solution in beamforming [12]. We present here a column-wise ${ }^{1}$ proportional solution for our model using autocorrelation matrices based on instantaneous channel matrices:

$$
\begin{aligned}
\mathbf{R}_{\mathbf{y}} & =\mathbf{H H}^{H}+\mathbf{H}_{B} \mathbf{H}_{B}^{H}+\sigma_{n}^{2} \mathbf{I}_{n_{B S}} \\
\mathbf{W}_{C} & =\mathbf{R}_{\mathbf{y}}^{-1} \mathbf{H}
\end{aligned}
$$

\section{B. Hybrid solutions}

Joint optimisation of the effective beamforming matrix AD under the constant modulus constraint is known to be a non-convex problem [8]. Therefore we consider a suboptimal scheme which consists firstly in extracting the weight's phases of the full digital solutions and use them in the analog stage. Secondly, into the digital stage, considering that we have more flexibility, we will always use the Capon solution calculated on the effective channel matrix. The following equations summerize the HBF solutions, where $i$ stands for ZF, GS or $\mathrm{C}$

\footnotetext{
${ }^{1}$ Since scalar factors of the beamforming columns do not change the SINR
} 
respectively for the zero forcing, Gram-Schmidt and the Capon solutions:

$$
\begin{aligned}
\mathbf{A}_{i} & =e^{j \arg \left(\mathbf{W}_{i}\right)} \\
\mathbf{H}_{e} & =\mathbf{A}_{i}^{H} \mathbf{H} \\
\mathbf{H}_{B, e} & =\mathbf{A}_{i}^{H} \mathbf{H}_{B} \\
\mathbf{R}_{\mathbf{y}}^{\prime} & =\mathbf{H}_{e} \mathbf{H}_{e}^{H}+\mathbf{H}_{B, e} \mathbf{H}_{B, e}^{H}+\sigma_{n}^{2} \mathbf{A}_{i}^{H} \mathbf{A}_{i} \\
\mathbf{D} & =\mathbf{R}_{\mathbf{y}}^{\prime-1} \mathbf{H}_{e}
\end{aligned}
$$

\section{PERFormanCE ANALYSis}

In this section we analyze the output SINR of the HBF scheme based on the algorithms presented in the previous section and compare them with the full digital scheme algorithms. We plot on the same figure three sub-figures: (a) the full digital SINR $\mathrm{p} \gamma_{F D}^{(k)}$, (b) the the intermediate hybrid analog SINR $\mathrm{p} \gamma_{A}^{(k)}$ so that we can visualize the introduced imperfections and (c) the hybrid (total i.e. analog and digital stages) SINR $\mathrm{p} \gamma_{D}^{(k)}$.

We use a single path uniform linear array (ULA) channel model, i.e. all the channel matrices columns are in the form $\mathbf{h}=\frac{h}{\sqrt{n_{B S}}}\left[1, e^{-j 2 \pi d \sin (\theta)} \ldots e^{-j\left(n_{B S}-1\right) 2 \pi d \sin (\theta)}\right] p^{T}$ where $d$ is the inter-elements spacing relative to the wavelength, $\theta$ the path AoA and $h$ the pathloss. We use the following parameters values $\mathrm{p} n_{B S}=16, n_{R F}=n_{U}=$ 8 , the following arbitrary users AoAs for the simulation $\left\{-71^{\circ},-62.5^{\circ},-54^{\circ},-33^{\circ}, 5^{\circ}, 19^{\circ}, 34^{\circ}, 60^{\circ}\right\}$, a half wavelength inter-elements spacing $d=0.5$ and a variable blocker AoA in the range $\left[-90^{\circ}, 90^{\circ}\right]$.

The signal-to-noise ratio ( $\mathrm{SNR}$ ) is defined as $\mathrm{SNR}=1 / \sigma_{n}^{2}$ on an additive white gaussian noise channel. We are mainly interested in medium SNR regimes (between $10 \mathrm{~dB}$ and $20 \mathrm{~dB}$ ) and high blockers-to-users received power levels (blockers powers up to $30 \mathrm{~dB}$ higher than users received power).

\section{A. Users AoA effect}

In this section we compare the SINR of two users, one located near the endfire at $-71^{\circ}$ in fig.(3) and the other one near the broadside at $5^{\circ}$ in fig.(4). We notice an SINR drop around the user location for all algorithms in both schemes. This is due to the limited angular resolution of the array. The magnitude constraint operation widens the drop interval for all algorithms with the least deterioration for Capon algorithm. Furthermore it is well pknown that the resolution decreases with the scanning angle, this explains the wider drops for the near endfire user. Also secondary SINR drops appear due to the misplaced interference nulls introduced by the magnitude constraints. We notice that the digital stage corrects these drops almost to the maximal level of the full digital scheme, and corrects the resolution loss moderately.

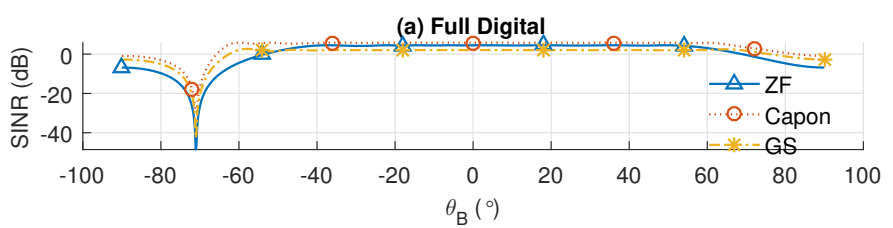

(b) Hybrid (Analog Stage)
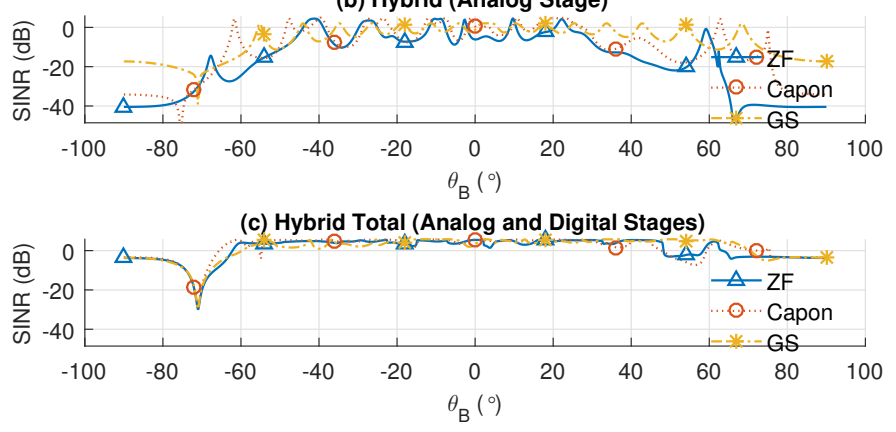

Fig. 3. SINR of the user located at $-71^{\circ}$, all users have a pathloss of $0 d B$ and the blocker a pathloss of $30 \mathrm{~dB}$.
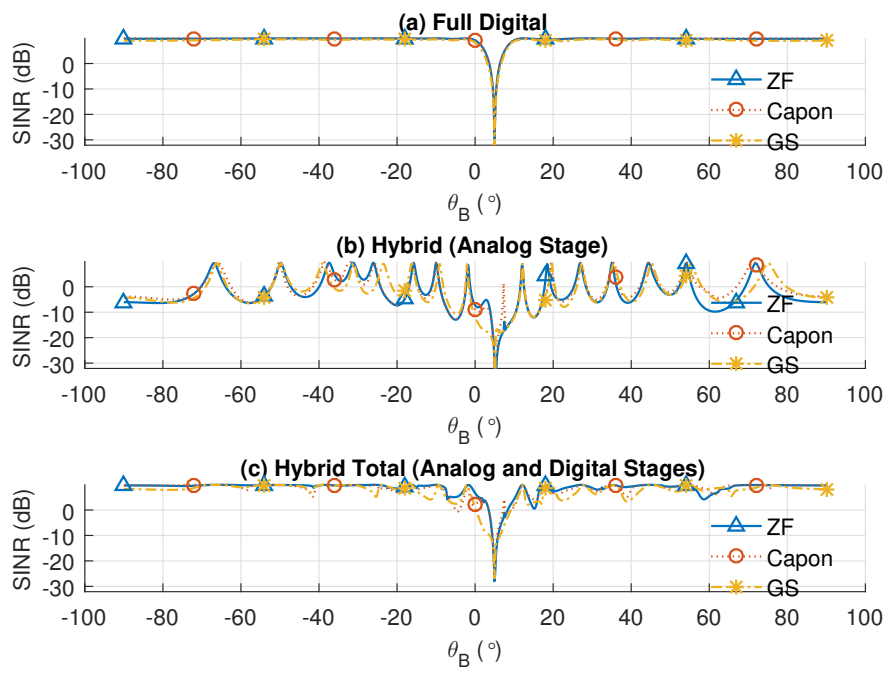

Fig. 4. SINR of the user located at $5^{\circ}$, all users have a pathloss of $0 d B$ and the blocker a pathloss of $30 \mathrm{~dB}$.

\section{B. Blocker power effect}

In this section we compare the SINR of the user located at $34^{\circ}$ in fig.(5), fig.(6) and fig.(7) for blockers levels of $h=0 d B$ $h=+30 d B h=+60 d B$ with an SNR $=20 d B$. We notice that the secondary drops introduced in the analog stage appear to be more severe as the blocker level increases, this is due to the decreased interference rejection of this stage which passes from a very high value in the full digital case ( $\infty$ for the ZF) to a lesser amount that could attain the side lobe levels of $-13 d B$ in the worst case. For all that the digital stage is still able to correct this loss with a slight dependence on the blocker level.

\section{Conclusion}

In this paper we have examined the SINR of different HBF solutions for SCs, constructed with a simple scheme which 

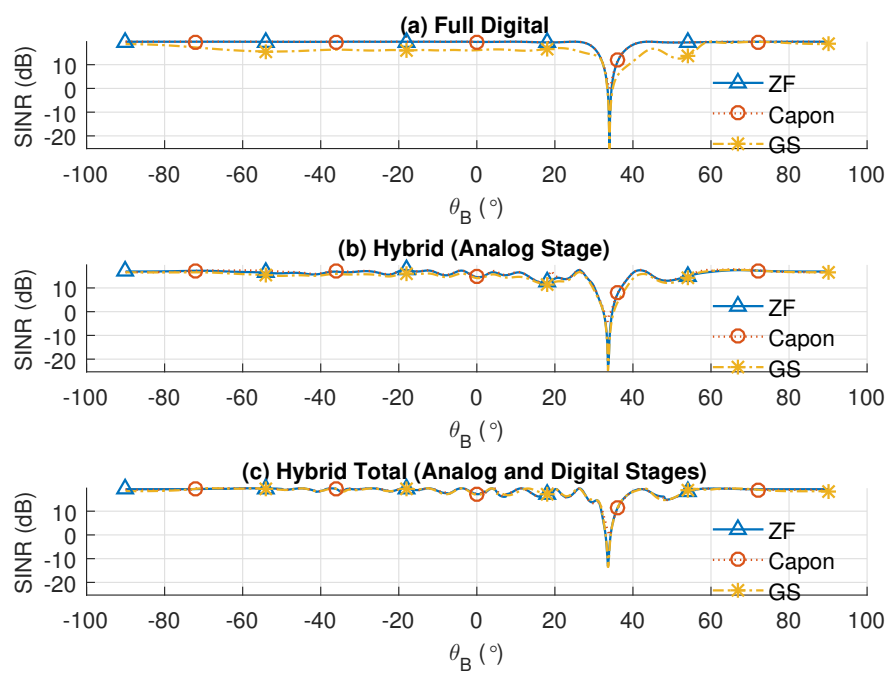

Fig. 5. SINR of the user located at $34^{\circ}$, all users have a pathloss of $0 d B$ and the blocker a pathloss of $0 d B$.

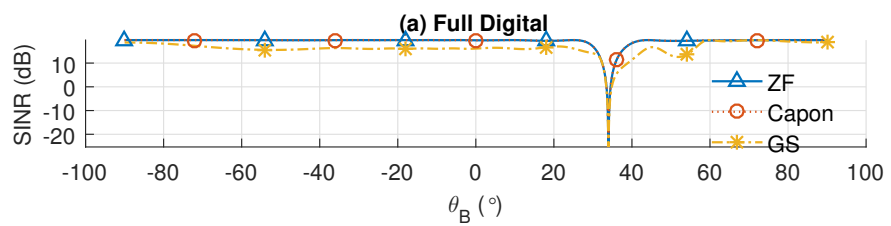

(b) Hybrid (Analog Stage)
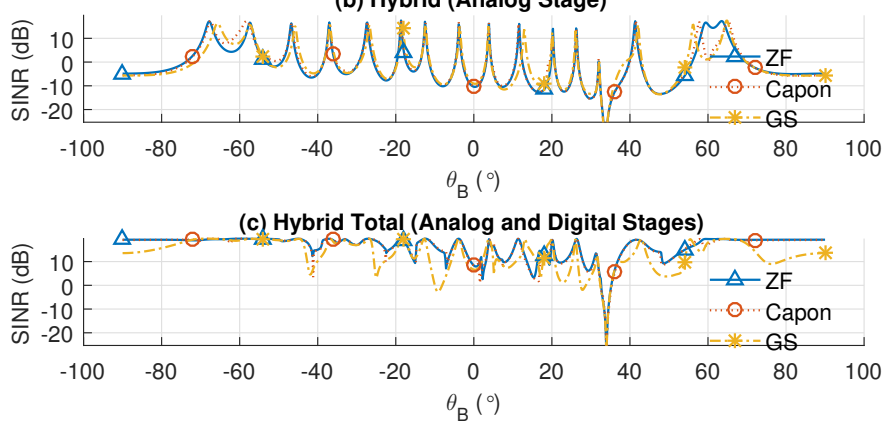

Fig. 6. SINR of the user located at $34^{\circ}$, all users have a pathloss of $0 d B$ and the blocker a pathloss of $+30 d B$.

consisted in using known full digital algorithms weights phases. Reduced number of antennas and RF chains reasonable for $\mathrm{cmW}$ bands have been envisaged. We showed that in a multiuser scenario with high blockers levels (between 0 to $60 \mathrm{~dB}$ higher than users) the suboptimal operation of using plain full digital weights phases with constant modulus in the analog stage introduces different losses that degrade with users AoAs and blockers levels. These losses are generally compensated by the Capon based digital stage with slight dependence on the analog stage algorithm. In future works performances analysis will be extended to multi-path channel models, limited resolution phase shifters, uniform circular arrays to relieve the spatial resolution dependency and limited ADCs resolution to model quantization noise and limited dynamic range.

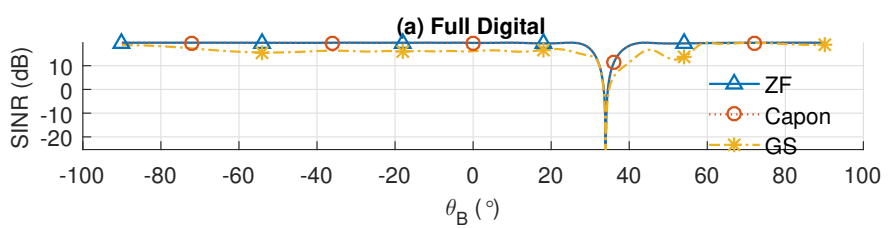

(b) Hybrid (Analog Stage)
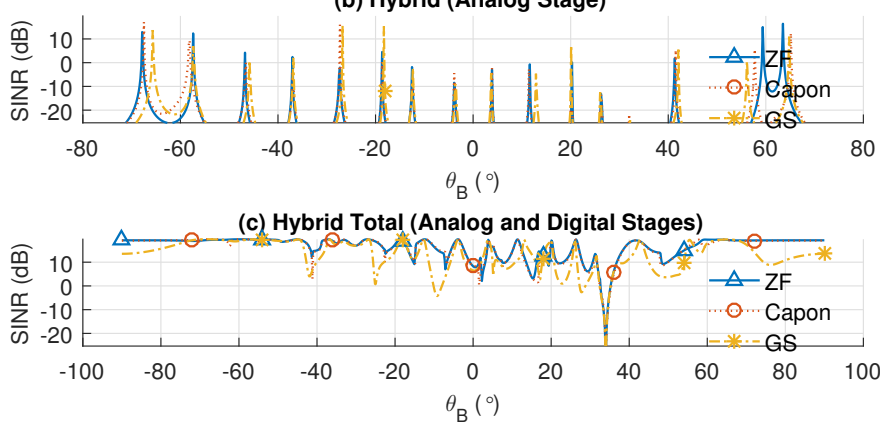

Fig. 7. SINR of the user located at $34^{\circ}$, all users have a pathloss of $0 d B$ and the blocker a pathloss of $+60 d B$.

\section{REFERENCES}

[1] T. Levanen, J. Venalainen, and M. Valkama, "Interference Analysis and Performance Evaluation of 5g Flexible-TDD Based Dense SmallCell System," in 2015 IEEE 82nd Vehicular Technology Conference (VTC2015-Fall), Sep. 2015, pp. 1-7.

[2] J. Shapira, "Microcell engineering in cdma cellular networks," IEEE Transactions on Vehicular Technology, vol. 43, no. 4, pp. 817-825, Nov 1994.

[3] A. F. Molisch, V. V. Ratnam, S. Han, Z. Li, S. L. H. Nguyen, L. Li, and K. Haneda, "Hybrid Beamforming for Massive MIMO: A Survey," IEEE Communications Magazine, vol. 55, no. 9, pp. 134-141, 2017.

[4] Y. Zhu and T. Yang, "Low Complexity Hybrid Beamforming for Uplink Multiuser mmWave MIMO Systems," in 2017 IEEE Wireless Communications and Networking Conference (WCNC), Mar. 2017, pp. 1-6.

[5] X. Zhang, M. Coldrey, T. Eriksson, and M. Viberg, "Hybrid beamforming in uplink massive MIMO systems in the presence of blockers," in 2017 IEEE International Conference on Acoustics, Speech and Signal Processing (ICASSP), Mar. 2017, pp. 6503-6507.

[6] Y.-C. Ko and M.-J. Kim, "Channel estimation and analog beam selection for uplink multiuser hybrid beamforming system," EURASIP Journal on Wireless Communications and Networking, vol. 2016, no. 1, Dec. 2016.

[7] S. Han, I. Chih-Lin, Z. Xu, and C. Rowell, "Large-scale antenna systems with hybrid analog and digital beamforming for millimeter wave $5 \mathrm{~g}$," IEEE Communications Magazine, vol. 53, no. 1, pp. 186-194, 2015.

[8] J. Li, L. Xiao, X. Xu, and S. Zhou, "Robust and Low Complexity Hybrid Beamforming for Uplink Multiuser MmWave MIMO Systems," IEEE Communications Letters, vol. 20, no. 6, pp. 1140-1143, Jun. 2016.

[9] J. Mo, A. Alkhateeb, S. Abu-Surra, and R. W. Heath, "Hybrid Architectures With Few-Bit ADC Receivers: Achievable Rates and EnergyRate Tradeoffs," IEEE Transactions on Wireless Communications, vol. 16, no. 4, pp. 2274-2287, Apr. 2017.

[10] H. Q. Ngo, E. G. Larsson, and T. L. Marzetta, "Energy and Spectral Efficiency of Very Large Multiuser MIMO Systems," IEEE Transactions on Communications, vol. 61, no. 4, pp. 1436-1449, Apr. 2013.

[11] S. M. Kogon, "Eigenvectors, diagonal loading and white noise gain constraints for robust adaptive beamforming," in The Thrity-Seventh Asilomar Conference on Signals, Systems Computers, 2003, vol. 2, Nov. 2003, pp. 1853-1857 Vol.2.

[12] A. Hassanien, S. Shahbazpanahi, and A. B. Gershman, "A generalized capon estimator for localization of multiple spread sources," IEEE Transactions on Signal Processing, vol. 52, no. 1, pp. 280-283, Jan. 2004. 